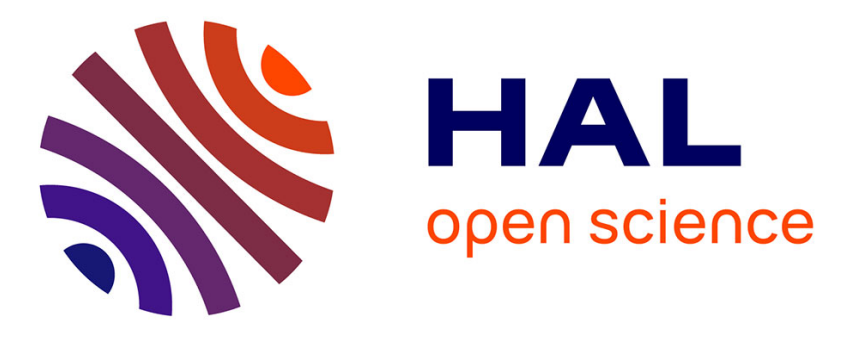

\title{
Object-level Impedance Control for Dexterous Manipulation with Contact Uncertainties using an LMI-based Approach
}

A Caldas, A Micaelli, M Grossard, M Makarov, Pedro Rodriguez-Ayerbe, D Dumur

\section{To cite this version:}

A Caldas, A Micaelli, M Grossard, M Makarov, Pedro Rodriguez-Ayerbe, et al.. Object-level Impedance Control for Dexterous Manipulation with Contact Uncertainties using an LMI-based Approach. 2015 IEEE International Conference on Robotics and Automation (ICRA), May 2015, Seattle, WA, United States. pp. 3668 - 3674, 10.1109/ICRA.2015.7139708 . hal-01138206

\section{HAL Id: hal-01138206 \\ https://hal.science/hal-01138206}

Submitted on 1 Apr 2015

HAL is a multi-disciplinary open access archive for the deposit and dissemination of scientific research documents, whether they are published or not. The documents may come from teaching and research institutions in France or abroad, or from public or private research centers.
L'archive ouverte pluridisciplinaire HAL, est destinée au dépôt et à la diffusion de documents scientifiques de niveau recherche, publiés ou non, émanant des établissements d'enseignement et de recherche français ou étrangers, des laboratoires publics ou privés. 


\title{
Object-level Impedance Control for Dexterous Manipulation with Contact Uncertainties using an LMI-based Approach
}

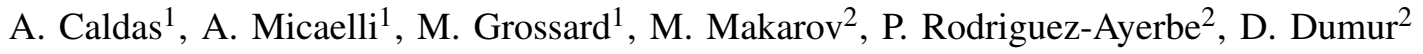

\begin{abstract}
This paper presents a new control scheme for dexterous manipulation of an object by a multifingered hand. The effects of the uncertainties on the contact orientation and location are investigated and taken into account in the control design, allowing to manipulate the object robustly. The control law has three main objectives: (i) ensuring the motion control of the object, (ii) satisfying the constraints of the manipulation system, and (iii) being robust to the uncertainties on the contact point. The proposed control is based on a state feedback architecture with robust pole placement by an LMI approach. The controller is designed offline and can be related to an object-level impedance controller. The constraints of the manipulation system, e.g. the friction constraints, are taken into account with an additional control action, based on an online LMI evaluation. Simulation results are presented and demonstrate that the proposed control law ensures the three main objectives.
\end{abstract}

\section{INTRODUCTION}

In the last few decades, robotics has evolved into ever more complex systems working on ever more complex manipulation tasks. From these emerging needs, roboticists have proposed new mechanical designs and multifingered hands have been developped. Various strategies have been proposed to control these mechanical systems and the field of dexterous manipulation is still an active field of research [1]-[3].

One difficulty in dexterous manipulation tasks is to deal with uncertainties. In the present state of technology, a perfect geometric representation of a scene is very difficult to achieve and needs various sensor feedbacks [4]. For instance, tactile and force sensors with a high $3 \mathrm{D}$ resolution are still very expensive and difficult to use in practice due to mechanical integration problems. Therefore, to better comply with practical experimental conditions, control laws for dexterous manipulation should include considerations about geometric uncertainties on the contact point. Two different approaches have been proposed in the literature to address this problem: identifying the contact point location or taking into account the uncertainties in the control scheme.

In the first approach, the contact point location can be deduced from the kinematic of the robotic hand by observing

\footnotetext{
${ }^{1}$ A. Caldas and M. Grossard are with the Interactive Robotic Laboratory of CEA-LIST and A. Micaelli is with the Interactive Simulation Laboratory of CEA-LIST 91192 Gif sur Yvette (e-mail: alex.caldas@cea.fr; alain.micaelli@cea.fr; mathieu.grossard@cea.fr).

${ }^{2}$ M. Makarov, P. Rodriguez-Ayerbe and D. Dumur are with Laboratoire des Signaux et Systemes (L2S, UMR CNRS 8506) CentraleSupelec - CNRS - Universite Paris-Sud, 3, rue Joliot Curie, 91192, Gif-sur-Yvette,France (e-mail: maria.makarov@ supelec.fr; pedro.rodriguez@supelec.fr; didier. dumur@supelec.fr).
}

the constrained motion of fingers [5]. With geometric considerations and using only joint angles and torque sensors, an algorithm was proposed to gather contact data while interacting with an unknown object [6]. Others methods use the self-posture changeability to determine the contact points [7]. Most of these methods are based on assumptions about the geometry of the system, which should be perfectly known.

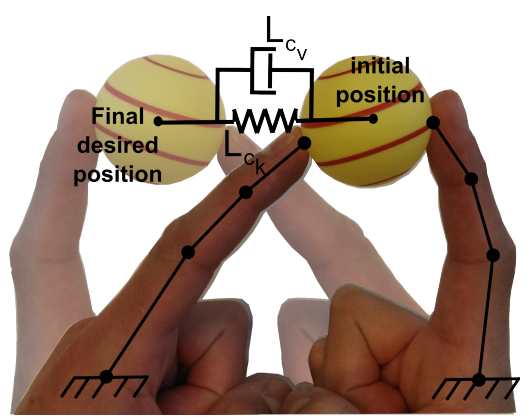

Fig. 1. Impedance behavior at the object-level

In the second approach, the uncertainties are explicitly defined in the system modeling, and a robust controller can be designed. Just a few studies have considered this framework. In [8] and [9], force and gravity regressors are proposed to cope with Jacobian uncertainties, and stability is studied with a Lyapunov function. An adaptive force/position control for a robot finger under surface kinematic uncertainties is proposed in [10]. However, the proposed controller assumes that a force/tactile sensor is used to provide measurements of normal forces.

Many problems dealing with robust control theory can be reduced to standard convex optimization problems involving Linear Matrix Inequalities (LMIs) [11]. Ensuring force-closure grasping can be formulated as an optimization problem with LMIs defining the friction constraints [12] and the grasp quality can be evaluated with LMIs adding bounded contact forces [13]. However, to the best of our knowledge, LMIs have not yet been employed for controller design in the dexterous manipulation field, and more specifically when contact uncertainties are taken into account, even though it is an interesting tool to develop robust control laws for robotic hands.

The aim of the present paper is to propose a new control strategy for dexterous manipulation without force/tactile sensor and dealing with uncertainties on the contact point location when the geometry of the system is not supposed perfectly known. The controller can be decomposed into two 
parts: (i) a state-feedback with pole placement, and (ii) an adaptive control action ensuring the constraints, computed via an LMI optimization. The proposed strategy is based on a linearized model and provides a framework to easily calculate an impedance controller under uncertainties (Fig. 1), with three main objectives: motion control, constraint validation (friction cone, unilaterality and boundaries of the contact forces), and robustness to contact uncertainties.

The paper is organized as follows: in section II, a statespace representation of the system is presented. Section III describes the proposed control scheme. Numerical examples and simulations of the controller are presented in section IV. Section $\mathrm{V}$ gives the conclusions and perspectives.

\section{MULTIFINGERED HAND REPRESENTATION}

This part presents the multifingered hand model, based on the screw theory [14], and introduces useful notations.

\section{A. State-Space Formulation}

The multifingered hand model is obtained by combining the dynamics of the fingers and the object:

$\left\{\begin{array}{l}M_{h}(q) \ddot{q}+C_{h}(q, \dot{q}) \dot{q}+N_{h}(q, \dot{q})-J_{h}\left(q, x_{o}\right)^{T} f_{c}=\tau \\ M_{o}\left(x_{o}\right) \ddot{x}_{o}+C_{o}\left(x_{o}, \dot{x}_{o}\right) \dot{x}_{o}+N_{o}\left(x_{o}, \dot{x}_{o}\right)+G f_{c}=0\end{array}\right.$

- For the first equation, $q=\left[\begin{array}{llll}q_{f_{1}} & q_{f_{2}} & \ldots & q_{f_{k}}\end{array}\right]^{T} \in \mathbb{R}^{n_{q}}$ is the actuated joint position vector for the $k$ fingers of the hand, $q_{f_{i}} \in \mathbb{R}^{n_{f_{i}}}$ being the actuated joint positions of the finger $i, \tau \in \mathbb{R}^{n_{q}}$ is the corresponding torque vector, and $M_{h}, C_{h}$ and $N_{h}$ are respectively the stacked inertia matrix, Coriolis, centrifugal and viscous friction matrix and gravity vector of the $k$ fingers of the hand, $J_{h} \in$ $\mathbb{R}^{n_{c} \times n_{q}}$ the hand Jacobian (with $n_{c}$ the dimension of the contact frame), and $f_{c} \in \mathbb{R}^{n_{c}}$ the contact forces applied on the object.

- For the second equation, $x_{o} \in \mathbb{R}^{n_{o}}$ a local coordinate of the object, with $n_{o}=6$ in $3 \mathrm{D}\left(n_{o}=3\right.$ in 2D) the dimension of the object coordinate, $M_{o} \in \mathbb{R}^{n_{o} \times n_{o}}$ the inertia matrix, $C_{o} \in \mathbb{R}^{n_{o} \times n_{o}}$ the Coriolis and centrifugal matrix, $N_{o} \in \mathbb{R}^{n_{o}}$ the gravity vector, $G \in \mathbb{R}^{n_{o} \times n_{c}}$ the grasp map relating the contact frames to the object frame.

The grasping of the object by the fingers is defined by the following constraint:

$$
J_{h}\left(q, x_{o}\right) \dot{q}=G^{T} \dot{x}_{o}
$$

According to (1)-(2), the system motion can be formulated at the object level as follows (see [14] for more details):

$$
M\left(q, x_{o}\right) \ddot{x}_{o}+C\left(q, x_{o}, \dot{q}, \dot{x}_{o}\right) \dot{x}_{o}+N\left(q, x_{o}, \dot{q}, \dot{x}_{o}\right)=G J_{h}^{-T} \tau
$$

with:

$$
\begin{aligned}
& M=M_{o}+G J_{h}^{-T} M_{h} J_{h}^{-1} G^{T} \\
& C=C_{o}+G J_{h}^{-T} C_{h} J_{h}^{-1} G^{T}+G J_{h}^{-T} M_{h} \frac{d}{d t}\left(J_{h}^{-1} G^{T}\right) \\
& N=N_{o}+G J_{h}^{-T} N_{h}
\end{aligned}
$$

In the following, the dependency on $q, \dot{q}, x_{o}, \dot{x}_{o}$ is dropped in the notations.
(2)-(6) are valid under the following assumptions:

A1 The system is not redundant, i.e. there is no internal movement of the fingers for a fixed position of the object. In this case, the hand Jacobian $J_{h}$ is square and invertible. This assumption could be relaxed with a model taking into account the joint redundancy [14][15].

A2 The contact points are fixed, which imply that $G$ is constant. This assumption is naturally made with (2), and will be relaxed later by taking into consideration the uncertainties on the contact point location.

A3 The contact forces remain in the friction cone. This constraint, which depends on the friction coefficient $\mu$, can be linearized [16] and expressed by the inequality:

$$
\Lambda f_{c}<\beta
$$

with $\Lambda \in \mathbb{R}^{n_{\text {constraints }} \times n_{c}}$ and $\beta \in \mathbb{R}^{n_{\text {constraints }} \text {, and } n_{\text {constraints }}}$ the number of constraints. This requirement must be satisfied by the control law.

A4 The grasp is manipulable, i.e. the desired motion can be generated by the fingers. In this case, the hand Jacobian is full row rank [14].

A5 The influence of gravity $N$ is negligible or compensated.

The linearization of (3) around an equilibrium point $\left[\begin{array}{ll}x_{o_{e q}} & \mathbb{O}_{n_{o}}\end{array}\right]^{T}$ leads to an LTI state-space representation:

$$
\frac{d}{d t}\left[\begin{array}{c}
x_{o} \\
\dot{x}_{o}
\end{array}\right]=\left[\begin{array}{cc}
\mathbb{O}_{n_{o} \times n_{o}} & \mathbb{I}_{n_{o} \times n_{o}} \\
\mathbb{O}_{n_{o} \times n_{o}} & -M_{e q}^{-1} C_{e q}
\end{array}\right]\left[\begin{array}{c}
x_{o} \\
\dot{x}_{o}
\end{array}\right]+\left[\begin{array}{c}
\mathbb{O}_{n_{o} \times n_{q}} \\
M_{e q}^{-1} G J_{h_{e q}}^{-T}
\end{array}\right] \tau
$$

where constant matrices $M_{e q}, C_{e q}$ and $J_{h_{e q}}$ define respectively the inertia matrix, Coriolis and centrifugal matrix, and the hand Jacobian calculated at the equilibrium point. In the remaining of the article, the model-based control design will be related to (8).

Furthermore, the contact forces can be expressed as a function of state $\left[\begin{array}{ll}x_{o} & \dot{x}_{o}\end{array}\right]^{T}$, by reformulating the object dynamics according to the previous assumptions:

$$
M_{o} \ddot{x}_{o}+C_{o} \dot{x}_{o}+G f_{c}=0 \Leftrightarrow G f_{c}=\left[\begin{array}{ll}
-C_{o} & -M_{o}
\end{array}\right]\left[\begin{array}{c}
\dot{x}_{o} \\
\ddot{x}_{o}
\end{array}\right]
$$

Using (8) and applying the pseudo-inverse of $G, f_{c}$ can be isolated in (9):

$$
\begin{aligned}
f_{c}= & G^{+}\left[\begin{array}{ll}
\mathbb{O}_{n_{o} \times n_{o}} & -C_{o}+M_{o} M_{e q}^{-1} C_{e q}
\end{array}\right]\left[\begin{array}{c}
x_{o} \\
\dot{x}_{o}
\end{array}\right] \\
& -G^{+} M_{o} M_{e q}^{-1} G J_{h_{e q}}^{-T} \tau+N_{G} \lambda
\end{aligned}
$$

with $(.)^{+}$defining the pseudo-inverse, which is not unique for the grasp map $G$. The term $N_{G} \lambda \in \operatorname{Ker}(G)$, with $N_{G}$ a base of the null space of $G$, is introduced, defining the non-uniqueness of the pseudo-inverse, and introducing a new degree of freedom $\lambda$, that will be used in the control law, and corresponding to the magnitude of the internal forces. 


\section{B. Model of the Contact Uncertainties}

Uncertainties on the contact point are due to modeling errors that can be of three types:

- Error on the contact surface (Fig. 2.a). This error occurs when the geometry of the object is not well known or is simplified (for calculation purposes). The contact frame is affected by an orientation uncertainty that would affect the grasp map $G$.

- Error on the object location (Fig. 2.b). This error occurs when the object position is uncertainly known, possibly due to a non valid initialization or a badly calibrated camera when visual servoing is used. The object frame considered in the control algorithm is affected by an offset compared to the real object frame. The grasp map $G$ is affected by this error.

- Fixed contact point assumption error, e.g. when rolling or soft finger contact occurs. In the previous section, the model assumes a fixed contact point. If in practice the contact is ensured by a surface or is subject to rolling, the equivalent contact point is unknown. This error also affects the grasp map $G$.

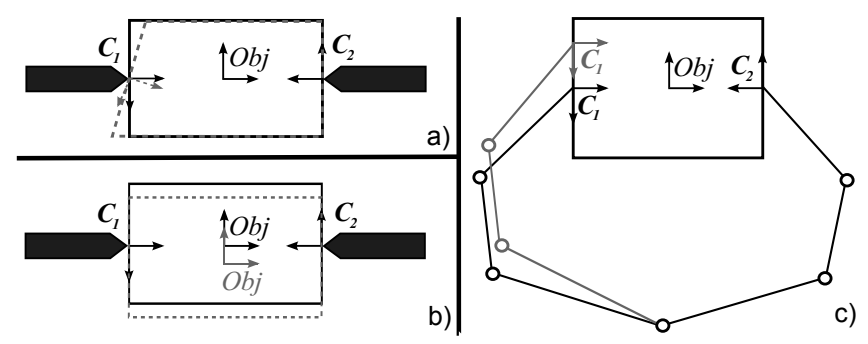

Fig. 2. Origins of uncertainties.

Let us note that other modeling errors can occur, e.g. error on the surface type, implying an uncertainty on the friction coefficient $\mu$, or error on the hand Jacobian $J_{h}$ (Fig. 2.c), that can affect the grasp map $G$ if the considered contact point is deduced from the inaccurate hand models. These errors are not taken into consideration in this paper, but the robustness of the controller to hand Jacobian uncertainties will be investigated in the examples.

The effects of the geometric uncertainties on the contact point are summarized by the following notation of the grasp map $G$, which can be defined as a function of uncertainties:

$$
G=G(\delta)
$$

with $\delta \in P_{\delta}$ the vector of uncertainties. $\delta$ can be structured as a translation and/or a rotation error, linear or nonlinear, with $P_{\delta}$ being the set of all the uncertainties considered in the system (linear dependence of $G$ on $\delta$ is investigated in [17]).

\section{CONTROL SCHEME}

\section{A. General Form}

The control inputs of the proposed state-space representation (8) are the joint torques. These signals can be expressed at the object level, allowing a direct connection between the object position error and the joint torques expressed at the object level. The transform between the object and the joint spaces depends on the hand Jacobian $J_{h}$ and the grasp map $G$, and can be expressed by:

$$
\tau=\underbrace{\hat{J}_{h_{\text {eq }}}^{T} G^{+} u}_{\tau_{\text {motion }}}+\underbrace{\hat{J}_{h_{e q}}^{T} N_{G} \lambda}_{\tau_{\text {internal }}}
$$

where $\hat{J}_{h_{e q}}$ is the estimate of the hand Jacobian at the equilibrium point, and $u$ the control input at the object level.

The transform $J_{h_{e q}}$ links the joint level to the contact level and the transform $G$ links the contact level to the object level. The first contribution $\tau_{\text {motion }}$ generates the object motion, expressed at the object level, and the second contribution $\tau_{\text {internal }}$ generates internal forces, which do not generate movement in the nominal case, but introduce an additional degree of freedom to fulfill the constraints. This decomposition of the torque vector is well known and was proposed since the 1980s [16]. While the proposed controller relies on this classical decomposition with a straightforward physical interpretation, the novelty of our contribution consists in the calculation of the signals $u$ and $\lambda$, which takes into account uncertainties with an LMI approach.

Injecting (12) in (8) leads to:

$$
\begin{aligned}
\frac{d}{d t}\left[\begin{array}{c}
x_{o} \\
\dot{x}_{o}
\end{array}\right]= & {\left[\begin{array}{cc}
\mathbb{O}_{n_{o} \times n_{o}} & \mathbb{I}_{n_{o} \times n_{o}} \\
\mathbb{O}_{n_{o} \times n_{o}} & -M_{e q}^{-1} C_{e q}
\end{array}\right]\left[\begin{array}{c}
x_{o} \\
\dot{x}_{o}
\end{array}\right] } \\
& +\left[\begin{array}{c}
\mathbb{O}_{n_{o} \times n_{q}} \\
M_{e q}^{-1} G J_{h_{e q}}^{-T}
\end{array}\right] \hat{J}_{h_{e q}}^{T} G^{+} u+\left[\begin{array}{c}
\mathbb{O}_{n_{o} \times n_{q}} \\
M_{e q}^{-1} G J_{h_{e q}}^{-T}
\end{array}\right] \hat{J}_{h_{e q}}^{T} N_{G} \lambda
\end{aligned}
$$

If the estimation of the hand Jacobian is perfect, i.e. $\hat{J}_{h_{e q}}=$ $J_{h_{e q}}$, the model becomes:

$$
\frac{d}{d t}\left[\begin{array}{c}
x_{o} \\
\dot{x}_{o}
\end{array}\right]=\underbrace{\left[\begin{array}{cc}
\mathbb{O}_{n_{o} \times n_{o}} & \mathbb{I}_{n_{o} \times n_{o}} \\
\mathbb{O}_{n_{o} \times n_{o}} & -M_{e q}^{-1} C_{e q}
\end{array}\right]}_{A}\left[\begin{array}{c}
x_{o} \\
\dot{x}_{o}
\end{array}\right]+\underbrace{\left[\begin{array}{c}
\mathbb{O}_{n_{o} \times n_{o}} \\
M_{e q}^{-1}
\end{array}\right]}_{B} u
$$

In this specific case, the object motion is only affected by the control input $u$.

Furthermore, combining (10) and (12) with a perfect estimation of the hand Jacobian yields:

$$
\begin{aligned}
f_{c}= & G^{+}\left[\begin{array}{ll}
\mathbb{O}_{n_{o} \times n_{o}} & -C_{o}+M_{o} M_{e q}^{-1} C_{e q}
\end{array}\right]\left[\begin{array}{l}
x_{o} \\
\dot{x}_{o}
\end{array}\right] \\
& -G^{+} M_{o} M_{e q}^{-1} u+N_{G} \lambda
\end{aligned}
$$

Thus, the contact forces can be written as:

$$
f_{c}=C_{f} x+B_{f} u+N_{G} \lambda
$$

with $x=\left[\begin{array}{ll}x_{o} & \dot{x}_{o}\end{array}\right]^{T}$ the state vector in (14).

Equations (14) and (16) will be used for the design of the robust control law.

\section{B. Objectives of the Control Law}

With respect to the proposed control input (12), the control objectives are:

- Motion tracking of the object. This objective is related to the control input $u$ which is defined explicitly in the 


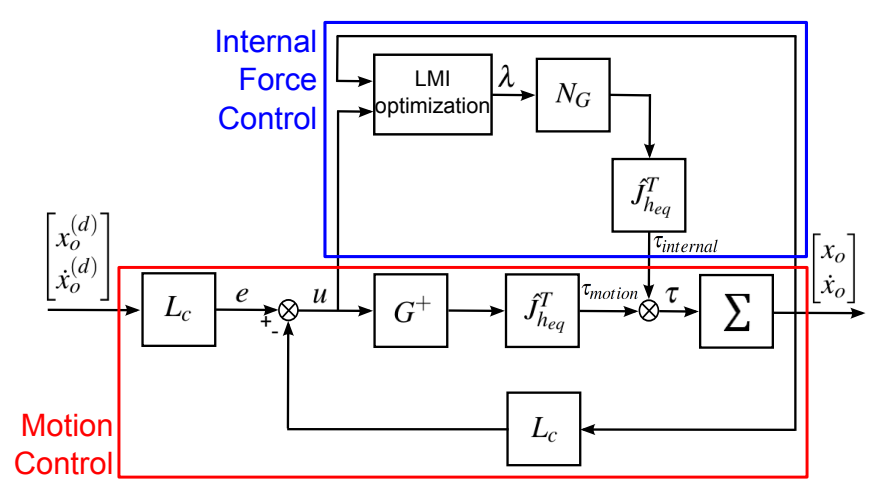

Fig. 3. General control scheme.

equation of motion (14). The fastest motion tracking will be searched, with the goal of reaching the desired final position, thus we focus on a regulation problem.

- Satisfying the system constraints, e.g. ensuring that the contact forces remain in the friction cone. This objective is achieved with the control input $\lambda$ that generates internal forces.

- The two previous objectives must be performed in presence of contact uncertainties. The proposed control design can be applied for a set of systems, which is equivalent to achieve the two previous objectives for the system affected by a set of uncertainties.

Another objective of the control design is the low complexity of the controller. In the view of the previous objectives, the control law will be a static feedback gain, equivalent to an impedance controller at the object level, and a dynamic action adding internal forces (Fig. 3).

\section{Impedance Motion Control}

As seen before, the motion control is ensured by the signal $u$. The proposed state feedback is:

$$
u=e-L_{c} x
$$

with $e$ the input of the closed-loop system and $L_{c}$ the state feedback matrix.

The closed-loop state-space equation becomes:

$$
\dot{x}=\left(A-B L_{c}\right) x+B e
$$

with $A$ and $B$ from (14).

The object motion dynamics can be specified by pole placement, i.e. by choosing the eigenvalues of the matrix $A-B L_{c}$. This can be achieved by defining a stable $\mathscr{D}$-region in the complex space. Classically, three different forms can be used to specify the stable $\mathscr{D}$-region [18]: the left halfplane, the cone and the disc (Fig. 4).

The $\mathscr{D}$-region can be described by LMI constraints depending on $P=P^{T} \succ 0$ a square positive-definite matrix (" $\succ$ " stands for positive definite), which defines the Lyapunov stability, $A$ the state matrix to stabilize, $Y=L_{c} P$ defining the state feedback, and the parameters $\alpha, r$ and $\theta$ defining respectively the left half-plane, the disc and the cone. The LMI problem consists in finding $P$ and $Y$ under the following constraints:
- The left half-plane defined by $\alpha$ :

$$
(A P-B Y)+\left(P A^{T}-Y^{T} B^{T}\right)+2 \alpha P \prec 0
$$

The choice of $\alpha>0$ avoids instability and sets minimal dynamics convergence.

- The disc centered in 0 with a radius $r$ :

$$
\left[\begin{array}{cc}
-r P & A P-B Y \\
P A^{T}-Y^{T} B^{T} & -r P
\end{array}\right] \prec 0
$$

The disc radius is chosen according to the maximal dynamics of the system, e.g. due to the hardware sampling period constraint or minimal noise influence.

- The cone centered in 0 with an angle $\theta$ (with $X^{\prime}=$ $A P-B Y)$ :

$$
\left[\begin{array}{cc}
\sin (\theta)\left(X^{\prime}+X^{\prime T}\right) & \cos (\theta)\left(X^{\prime}-X^{\prime T}\right) \\
\cos (\theta)\left(X^{\prime T}-X^{\prime}\right) & \sin (\theta)\left(X^{\prime}+X^{\prime T}\right)
\end{array}\right] \prec 0
$$

This sector allows to constrain the damping ratio.

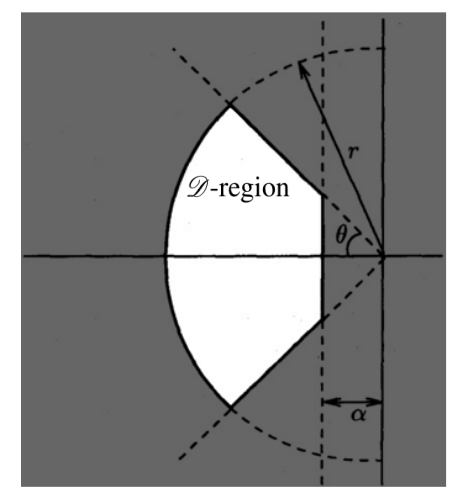

Fig. 4. LMI Regions [18].

The pole placement can be achieved by solving the feasability problem: finding $P$ and $Y$ such that (19) to (21) are true. Then, the state-feedback matrix is deduced:

$$
L_{c}=Y P^{-1}
$$

Moreover, in order to track the reference without steadystate error, a precompensation is calculated to define $e$ :

$$
e=L_{c} x^{(d)}
$$

with $x^{(d)}$ the desired state, i.e. the desired position and velocity of the object. The matrix $L_{c}$ can be decomposed as follows:

$$
L_{c}=\left[\begin{array}{ll}
L_{c_{k}} & L_{c_{v}}
\end{array}\right]
$$

where $L_{c_{k}}, L_{c_{v}} \in \mathbb{R}^{n_{o} \times n_{o}}$ define respectively the stiffness and the damping of the motion tracking at the object level, and the new object dynamics is deduced:

$$
M_{e q} \ddot{x}_{o}+\left(C_{e q}+L_{c_{v}}\right) \dot{x}_{o}+L_{c_{k}} x_{o}=e
$$

Note that (25) hightlights that this state feedback is equivalent to an impedance controller at the object level. 


\section{Internal Force Control}

The previous section provides a solution to the problem of motion control of the object, but the control law is relevant only if the constraints on the contact points are satisfied, particularly:

C1 The contact points are fixed and no rolling neither sliding are allowed. This condition will be relaxed in the next section, by taking into account the uncertainties in the contact points.

C2 The contact forces must remain in the friction cone.

C3 The contact forces are limited according to joint torque minimal $\tau_{\min }$ and maximal $\tau_{\max }$ limits.

These constraints can be satisfied by resolving an LMI problem on the contact forces.

The joint torque limits can be expressed at the contact level:

$$
\tau_{\min }<\tau<\tau_{\max }
$$

Introducing (1) and applying the assumptions specified in II-A:

$$
\tau_{\min }<M_{h} \ddot{q}+C_{h} \dot{q}-J_{h}^{T} f_{c}<\tau_{\max }
$$

The following inequalities are deduced:

$$
\begin{cases}J_{h}^{T} f_{c}+\left(-M_{h} \ddot{q}-C_{h} \dot{q}+\tau_{\text {min }}\right) & <0 \\ -J_{h}^{T} f_{c}+\left(M_{h} \ddot{q}+C_{h} \dot{q}-\tau_{\max }\right) & <0\end{cases}
$$

The expression of the contact forces (16) is introduced in the constraints (28) and (7):

$$
\begin{cases}\Lambda N_{G} \lambda+\Lambda\left(C_{f} x+B_{f} u\right)-\beta & <0 \\ J_{h}^{T} N_{G} \lambda+J_{h}^{T}\left(C_{f} x+B_{f} u\right)+\beta_{2}(q) & <0 \\ -J_{h}^{T} N_{G} \lambda-J_{h}^{T}\left(C_{f} x+B_{f} u\right)+\beta_{2}^{\prime}(q) & <0\end{cases}
$$

with $\beta_{2}(q)=-M_{h} \ddot{q}-C_{h} \dot{q}+\tau_{\min }$ and $\beta_{2}^{\prime}(q)=M_{h} \ddot{q}+C_{h} \dot{q}-$ $\tau_{\text {max }}$. The degree of freedom $\lambda$ allows one to fulfill these three constraints depending on the current state $x$, joint angles $q$ and control signals $u$. This problem can be solved by an online optimization with LMI constraints. The three constraints (29) can be formulated under an LMI form, by transforming the column of the matrix $\Lambda N_{G}, J_{h}^{T} N_{G}$ and $-J_{h}^{T} N_{G}$ and diagonalizing the vectors $\Lambda\left(C_{f} x+B_{f} u\right)-\beta$, $J_{h}^{T}\left(C_{f} x+B_{f} u\right)+\beta_{2}(q)$ and $-J_{h}^{T}\left(C_{f} x+B_{f} u\right)+\beta_{2}^{\prime}(q)$ (see [13] for more details).

Furthermore, robustness to external wrench disturbances applied on the object can be ensured by introducing an offset on $\lambda$.

\section{E. Algorithm Coping with Uncertainties}

If uncertainties affected the system linearly, the classical method could have been to write it in an Linear Parametric Varying (LPV) form. Here, the uncertainties on the contact points affect the grasp map, and the system representation (14) is not linear according to the matrix $G$, thus it is not straightforward to formulate the model as a LPV system. Instead, the following approach is used.

A set $P_{\Sigma}$ of all the possible state-space representations $\Sigma=(A, B, C, D)$ depending on the uncertainties is defined:

$$
\Sigma \in P_{\Sigma} \Leftrightarrow\left\{\forall \delta \in P_{\delta}, \Sigma:=\{\dot{x}=A(\delta) x+B(\delta) u\}\right\}
$$

Knowing that the uncertainties do not affect linearly this set, no assumption can be made about the convexity of $P_{\Sigma}$. The uncertainties can be taken into account by discretizing the polytope $P_{\delta}$, implying a discretization of $P_{\Sigma}$, and then test the LMI problems from III-C and III-D for each system resulting from the discretization of $P_{\Sigma}$ (this set of systems will be called thereafter discretized- $P_{\Sigma}$ systems).

For the motion control problem, and according to the motion control objectives defined in III-B, the proposed algorithm consists in minimizing the trace of $P$, which will maximize the eigenvalues real part of the closed-loop, and result in the fastest response. The problem is formulated as follows:

$$
\left\{\begin{array}{cc}
\min _{Y, P} \operatorname{Tr}(P) & \\
& \text { s.t. for all } \delta_{i} \in P_{\delta} \\
& (19)-(21) \text { are satisfied } \\
& \text { end for }
\end{array}\right.
$$

Others criteria can be found in the literature [19], e.g. imroving the robustness according to unstructured uncertainties.

The resulting static gain $L_{c}$ ensures the stability of every discretized- $P_{\Sigma}$ systems. If the sampling step of $P_{\Sigma}$ is small enough, the motion tracking is guaranteed even in case of uncertain contact point location, rolling contact or soft finger contact.

The internal forces are also deduced by solving the LMI problems deduced from the constraints (29) for every discretized-P $P_{\Sigma}$ systems. The overall stategy is depicted in Fig. 3 .

\section{NUMERICAL EXAMPLES AND SIMULATIONS}

This section presents the simulation results of the proposed control scheme for different examples.

\section{A. $2 D$ uncertain manipulation system}

The following 2D example relates to a grasp with two contact points. The contact forces $f_{c}=\left[f_{c_{1}} f_{c_{1 y}} f_{c_{2 x}} f_{c_{2 y}}\right]^{T} \in$ $\mathbb{R}^{4}$ have to satisfy the following Coulomb friction constraints (Fig. 5):

$$
\left(\begin{array}{cccc}
-1 & -\mu & 0 & 0 \\
1 & -\mu & 0 & 0 \\
0 & 0 & -1 & -\mu \\
0 & 0 & 1 & -\mu
\end{array}\right)\left(\begin{array}{l}
f_{c_{1 x}} \\
f_{c_{1 y}} \\
f_{c_{2 x}} \\
f_{c_{2 y}}
\end{array}\right)<\left(\begin{array}{l}
0 \\
0 \\
0 \\
0
\end{array}\right)
$$

with the friction coefficient $\mu=1$. The grasp map is:

$$
G_{0}=\left(\begin{array}{cccc}
0 & -1 & 0 & 1 \\
1 & 0 & -1 & 0 \\
r_{o} & 0 & r_{o} & 0
\end{array}\right)
$$

with the length of the rectangular object fixed to $r_{o}=20 \mathrm{~mm}$. Two types of uncertainties are considered:

E1 Translation error (Fig. 5a). The direction of the contact force is considered as known, but the location of the contact point is uncertain. The grasp map becomes:

$$
G_{r}=\left(\begin{array}{cccc}
0 & -1 & 0 & 1 \\
1 & 0 & -1 & 0 \\
r_{o} & 0 & r_{o} & \delta
\end{array}\right)
$$


where $\delta= \pm 5 \mathrm{~mm}$ represents the translation uncertainty of the second contact point.

E2 Orientation error (Fig. 5b). The location of the contact point is considered as known but the orientation of the contact force is uncertain. The grasp map becomes:

$$
G_{r}=\left(\begin{array}{cccc}
0 & -1 & \sin \left(\theta_{\delta}\right) & \cos \left(\theta_{\delta}\right) \\
1 & 0 & \cos \left(\theta_{\delta}\right) & \sin \left(\theta_{\delta}\right) \\
r_{o} & 0 & r_{o} \cos \left(\theta_{\delta}\right) & -r_{o} \sin \left(\theta_{\delta}\right)
\end{array}\right)
$$

with $\theta_{\delta}= \pm 10^{\circ}$ being the orientation error of the second contact point.

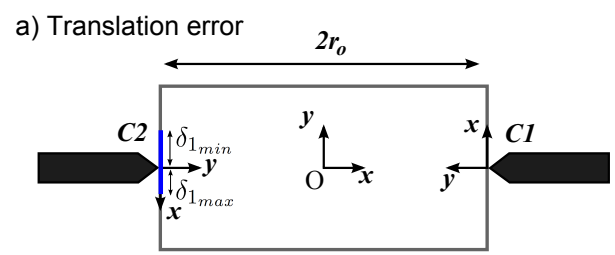

b) Rotation error

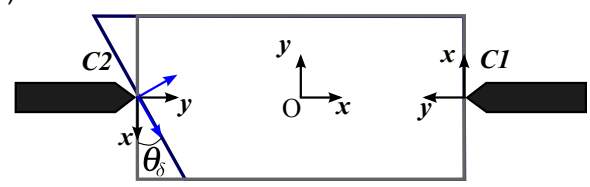

Fig. 5. Proposed example: a) translation error and b) rotation error

\section{B. Results}

The motion control was designed with the following LMI parameters:

- $\alpha=0.5$, which ensures $\operatorname{Re}($ pole $)<-0.5$, providing a stability constraints and minimal dynamics.

- $\theta=25$, which guarantees a damping ratio of $\xi \simeq 0.6$.

- $r=7$, which sets the maximal dynamics.

The algorithm (31) (called thereafter Fast Controller) was tested for the motion control and compared with another feasible controller designed without trace minimization (called thereafter Feasible Controller), i.e. in the previously defined LMI region. The LMI-based optimization algorithm was applied for the two examples (E1-E2). The motion consists in placing the object on the right with a different orientation (Fig. 7.a-c and 8).

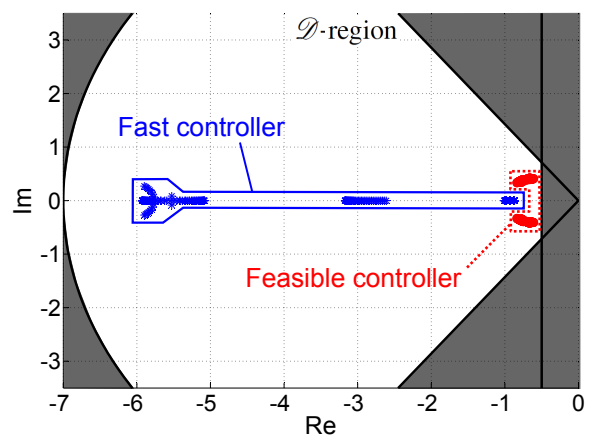

Fig. 6. Closed-loop poles for all the discretized- $P_{\Sigma}$ systems with $0.5 \mathrm{~mm}$ step on $\delta$
The following observations can be made:

- The poles of all the discretized-P $P_{\Sigma}$ systems are in the $\mathscr{D}$ region (Results are presented for example E1 in Fig. 6) for the Fast and Feasible Controllers. The fast behavior is obtained by placing the poles on the left side of the LMI region.

- The static error is negligible in the two examples (Fig.7.a-c and 8), whatever the chosen behavior (fast or feasible) is, and when no external disturbance is applied. The control law is therefore robust to model uncertainties: recall that an estimation of the nominal hand Jacobian $\hat{J}_{h_{e} q}$ is used in the controller, while the true hand Jacobian $J_{h}\left(q, x_{o}\right)$ varies in the model during the simulation.

- Fast behavior is achieved. In Fig. 7.a-c, a faster response of the system is found with the Fast Controller than with the Feasible Controller.

- The magnitude of the control input is admissible (Fig. 7.d). This result is found by setting the circle LMI region, defining the maximal dynamics.
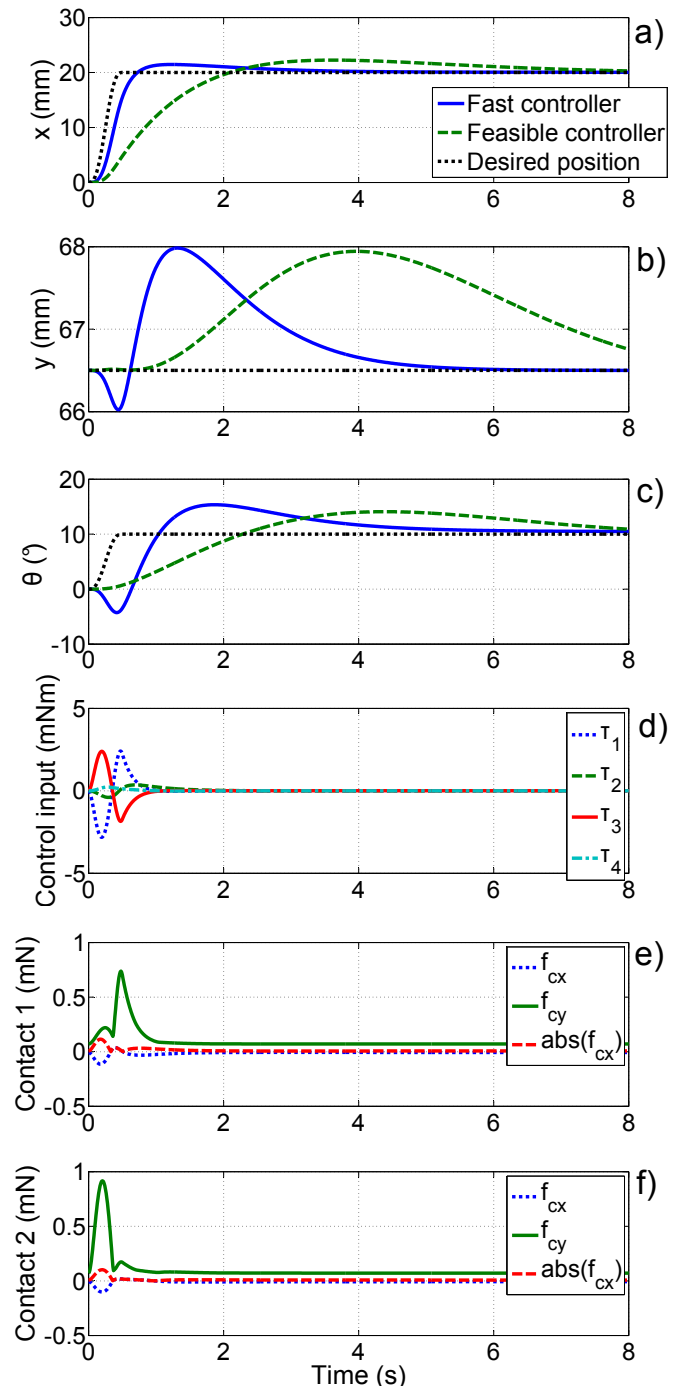

Fig. 7. Dynamic response (Example E1) 


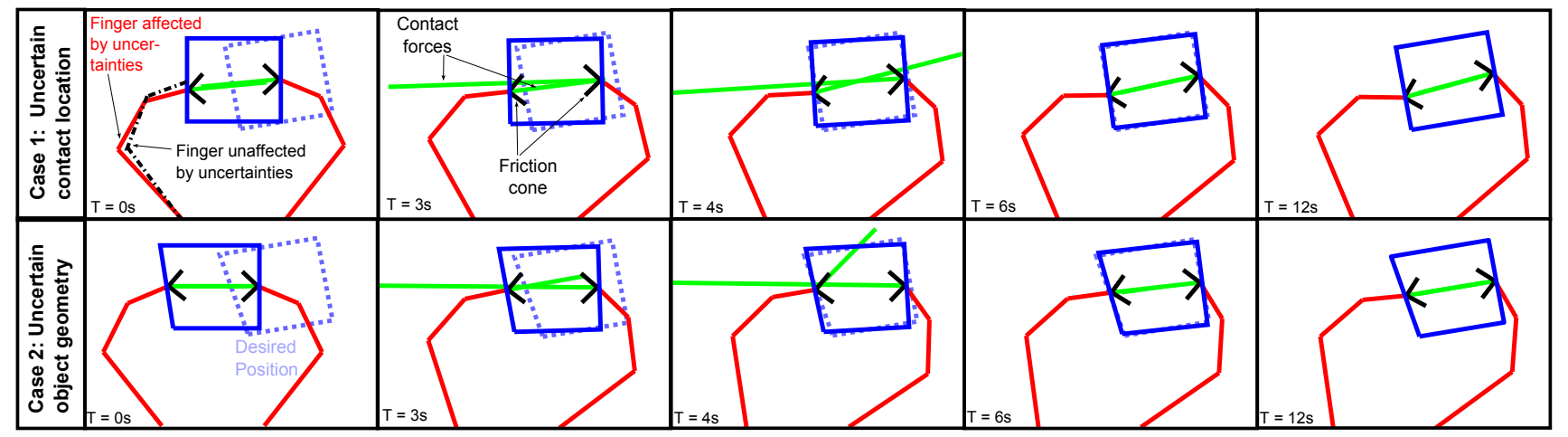

Fig. 8. Simulation.

- The contact forces remain in the friction cone during the movement (Fig. 8 and 7.e-f). The contact force on the $y$-axis is always positive, meaning that only unilateral forces can be applied, and $f_{c_{y}}$ is always higher than $\left|f_{c_{x}}\right|$, validating the cone constraint. An offset on $\lambda$, i.e. $f_{c_{y}}$, of $100 \mu \mathrm{N}$ was introduced to achieve robustness to external disturbances, e.g. an external wrench applied at the object level.

\section{CONCLUSIONS}

The paper presents a new control law to cope with contact uncertainties for dexterous manipulation with a multifingered hand. Three objectives are achieved: (i) motion tracking of the object without any static error, (ii) satisfaction of the system constraints, i.e. unilaterality of the contact forces, friction constraints, and bounded joint torques, (iii) robustness to contact uncertainties. The controller was tested on two cases, translation and rotation errors, the objectives were fulfilled and a robust behavior was observed.

The problem of the robustness to contact uncertainties addressed in this paper could be related to dexterous manipulation by a human without tactile sensing [20] [21]. It would be interesting to compare the proposed control scheme with the human control.

Next works will consider the non redundant case and 3D examples. Knowing that the algorithm is based on a linearized model, it would be interesting to evaluate the region of the workspace where the desired properties of the closed loop are guaranteed. Next step will be to implement and test the proposed controller in a practical case.

\section{REFERENCES}

[1] J. Martin and M. Grossard, "Design of a fully modular and backdrivable dexterous hand," The International Journal of Robotics Research (IJRR) Special Issue on 'Mechanics and Design of Robotic Hands', vol. 33, no. 5, pp. 783-798, 2014.

[2] A. Bicchi, "Hands for dexterous manipulation and robust grasping: A difficult road toward simplicity," IEEE Transactions on Robotics and Automation, vol. 16, no. 6, pp. 652-662, 2000.

[3] A. M. Okamura, N. Smaby, and M. R. Cutkosky, "An overview of dexterous manipulation," in IEEE International Conference on Robotics and Automation, 2000, vol. 1. IEEE, 2000, pp. 255-262.

[4] M. Grossard, G. Felippe, G. Hamon, and J. Martin, "Force sensing strategy for the backdrivable and dexterous cea hand," in International Conference on Advanced Robotics, 2013. IEEE, 2013, pp. 1-7.
[5] S. Haidacher and G. Hirzinger, "Contact point identification in multifingered grasps exploiting kinematic constraints," in IEEE International Conference on Robotics and Automation, vol. 2. IEEE, 2002, pp. 1597-1603.

[6] M. Huber and R. A. Grupen, "2-d contact detection and localization using proprioceptive information," IEEE Transactions on Robotics and Automation, vol. 10, no. 1, pp. 23-33, 1994.

[7] M. Kaneko and K. Tanie, "Contact point detection for grasping an unknown object using self-posture changeability," IEEE Transactions on Robotics and Automation, vol. 10, no. 3, pp. 355-367, 1994.

[8] C.-C. Cheah, H.-Y. Han, S. Kawamura, and S. Arimoto, "Grasping and position control for multi-fingered robot hands with uncertain jacobian matrices," in IEEE International Conference on Robotics and Automation, vol. 3. IEEE, 1998, pp. 2403-2408.

[9] C.-C. Cheah, C. Liu, and J. Slotine, "Adaptive jacobian tracking control of robots with uncertainties in kinematic, dynamic and actuator models," IEEE Transactions on Automatic Control, vol. 51, no. 6, pp. 1024-1029, 2006.

[10] Z. Doulgeri and Y. Karayiannidis, "Force position control for a robot finger with a soft tip and kinematic uncertainties," Robotics and Autonomous Systems, vol. 55, no. 4, pp. 328 - 336, 2007.

[11] S. P. Boyd, L. El Ghaoui, E. Feron, and V. Balakrishnan, Linear matrix inequalities in system and control theory. SIAM, 1994, vol. 15.

[12] M. Buss, H. Hashimoto, and J. B. Moore, "Dextrous hand grasping force optimization," IEEE Transactions on Robotics and Automation, vol. 12, no. 3, pp. 406-418, 1996.

[13] L. Han, J. C. Trinkle, and Z. X. Li, "Grasp analysis as linear matrix inequality problems," IEEE Transactions on Robotics and Automation, vol. 16 , no. 6, pp. 663-674, 2000.

[14] R. M. Murray and S. S. Sastry, A Mathematical Introduction to Robotic Manipulation. CRC press, 1994.

[15] A. Bicchi, M. Gabiccini, and M. Santello, "Modelling natural and artificial hands with synergies," Philosophical Transactions of the Royal Society B: Biological Sciences, vol. 366, no. 1581, pp. 31533161, 2011.

[16] J. Kerr and B. Roth, "Analysis of multifingered hands," The International Journal of Robotics Research, vol. 4, no. 4, pp. 3-17, 1986.

[17] A. Caldas, A. Micaelli, M. Grossard, M. Makarov, P. RodriguezAyerbe, and D. Dumur, "New metric for wrench space reachability of multifingered hand with contact uncertainties," in IEEE/ASME International Conference on Advanced Intelligent Mechatronics (AIM), July 2014, pp. 1236-1242.

[18] M. Chilali and P. Gahinet, "Hinf design with pole placement constraints: an LMI approach," IEEE Transactions on Automatic Control, vol. 41, no. 3, pp. 358-367, 1996.

[19] A. Luca, P. Rodriguez-Ayerbe, D. Dumur et al., "Control of disturbed LPV systems in a LMI setting," in 18th IFAC World Congress, Milan, Italy, 2011.

[20] J. Monzée, Y. Lamarre, and A. M. Smith, "The effects of digital anesthesia on force control using a precision grip," Journal of neurophysiology, vol. 89, no. 2, pp. 672-683, 2003.

[21] R. Johansson and G. Westling, "Signals in tactile afferents from the fingers eliciting adaptive motor responses during precision grip," Experimental Brain Research, vol. 66, no. 1, pp. 141-154, 1987. 